\title{
Embedding tiling spaces in surfaces
}

\author{
by
}

Charles Holton (Austin, TX) and Brian F. Martensen (Mankato, MN)

\begin{abstract}
We show that an aperiodic minimal tiling space with only finitely many asymptotic composants embeds in a surface if and only if it is the suspension of a symbolic interval exchange transformation (possibly with reversals). We give two necessary conditions for an aperiodic primitive substitution tiling space to embed in a surface. In the case of substitutions on two symbols our classification is nearly complete. The results characterize the codimension one hyperbolic attractors of surface diffeomorphisms in terms of asymptotic composants of substitutions.
\end{abstract}

1. Introduction. We are interested in orientable one-dimensional expanding hyperbolic attractors of diffeomorphisms. In particular, we want to know which of these occur as attractors of diffeomorphisms of surfaces. According to Williams' classification ([Wil67, Wil70]), each one-dimensional expanding hyperbolic attractor is the inverse limit of an expanding map on a bouquet of circles. As such they are either homogeneous solenoids or (one-dimensional) aperiodic primitive substitution tiling spaces. Homogeneous solenoids cannot embed in surfaces ([Fed80, CF02]), so we restrict our attention to substitution tiling spaces. An example of a substitution tiling space embedded in a surface may be found in [FHR03] and typifies the results here.

The classification of orientable one-dimensional expanding hyperbolic attractors of diffeomorphisms is complete in a sense. Homogeneous solenoids are distinguished by their cohomology. The Barge-Diamond invariant ([BD01]) for aperiodic primitive substitution tiling spaces is complete, but it is complicated and may not be algorithmically decidable.

One useful by-product of the Barge-Diamond machinery is a straightforward method for finding the inhomogeneities, the asymptotic composants

2000 Mathematics Subject Classification: Primary 37B50; Secondary 37C70, 37E35, 37B10, 37E05.

Key words and phrases: tiling spaces, pseudo-Anosov, surface embeddings, interval exchange transformations, free group automorphisms. 
in a substitution tiling space. This played a crucial role in [BDH03] and [CE03]. It shows up in this work as a verifiable necessary condition for a tiling space to embed in a surface. It turns out that if a tiling space embeds in a surface then it can be realized as the attractor of a diffeomorphism of a surface.

It is an important open problem in symbolic dynamics to determine which systems are the codings of interval exchanges ([Fog01, Rau79]). The question seems difficult, even when restricted to symbolic substitution systems. Going the other direction, it follows from the result of Boshernitzan and Carroll ([BC97]) that an interval exchange where all the lengths lie in the same quadratic extension of $\mathbb{Q}$ comes from a substitution; the authors point out that the converse is false. We present here some partial results about when a tiling space embeds in a surface, which addresses the question above because of the following:

THEOREM 1. Let $\Omega$ be a minimal shift space with finitely many asymptotic composants. Let $\mathcal{T}_{\Omega}$ be its associated tiling space. Then the shift map on $\Omega$ is flow equivalent to a symbolic interval exchange transformation (possibly with reversals) if and only if $\mathcal{T}_{\Omega}$ embeds in a surface.

Flow equivalence, rather than conjugacy, arises naturally here because symbolic morphisms (letter-to-word maps), as well as sliding block codes, preserve the structure of the associated tiling space. For a substitution, choosing the vector of tile lengths to be a left Perron eigenvector for the incidence matrix gives a geometric action of the substitution on the tiling space. For such a tiling space embedded in a surface, the geometric action of the substitution extends naturally to a "derived from pseudo-Anosov" diffeomorphism on the surface (or a related surface) having the embedded substitution tiling space as its unique codimension one attractor. Furthermore, every orientable one-dimensional expanding hyperbolic attractor for a surface diffeomorphism arises in this way.

Additional work has been done by Bruin ([Bru05]) regarding the computation of asymptotic arc-components for inverse limit spaces of a unimodal bonding map. Substitution tiling spaces appear as orientable double covers of these spaces and hence our results apply to these double covers. The rarity of being able to embed in a surface is well illustrated by applying our Corollary 3.2 to the table of examples and their asymptotic composants given in Figure 4 of that paper.

We show in Section 3 that the asymptotic composants in a tiling space are exactly the composants that bound the complement when the tiling space is embedded in a surface. (We thank M. Barge for suggesting this to us.) This gives a necessary condition for a tiling space to embed in a surface: The asymptotic composants must form cycles. For a primitive substitution that 
is a (positive) automorphism of the free group generated by its alphabet, this condition is sufficient as well. This is a consequence of the results of Bestvina and Handel ([BH92]). The cyclic fixed words in [BH92] are precisely the labelings of the cycles of asymptotics obtained here. Our tiling space embeds in the surface as the geometric lamination of the pseudo-Anosov diffeomorphism in [BH92] and the substitution acts as the automorphism on the homotopy of the surface. We emphasize that it is not necessary for the substitution to be an automorphism of a free group in order for an embedding to exist. It does follow from [BH92] that a substitution tiling space that embeds in a surface is homeomorphic to a substitution tiling space arising from a positive automorphism of a free group. Our work here implies an algorithm for finding such an automorphism given a generic substitution tiling space that embeds in a surface.

The proof of Theorem 1 is in Section 4. We first show that the lamination given by an essential embedding of a tiling space in a surface extends to a foliation (with singularities) of the surface. It follows that the asymptotic composants of a tiling space actually determine the surface for an essential embedding, when an embedding exists. The surface is readily identified by index theory.

We focus on substitutions on two symbols in Section 5. In this simple setting we classify the possible behaviors of the asymptotics. We show that an aperiodic tiling space generated by a primitive substitution on two symbols embeds in an orientable surface if and only if the substitution is Sturmian. This is related to Bestvina-Handel because a substitution on two symbols is Sturmian if and only if it is a positive automorphism on the free group generated by its alphabet.

2. Preliminaries. Let $\mathcal{A}$ be an alphabet, i.e., a finite set of symbols. Write $\mathcal{A}^{*}$ for the free monoid generated by $\mathcal{A}$. The elements of $\mathcal{A}^{*}$ are finite sequences in $\mathcal{A}$ regarded as words. We also consider one-sided infinite sequences and bi-infinite sequences in $\mathcal{A}$ to be words. A factor of a word is a finite contiguous subsequence. The terms prefix, suffix and length have their usual meanings. A subset $\mathcal{L}$ of $\mathcal{A}^{*}$ is called a language if: every factor of a word in $\mathcal{L}$ is again in $\mathcal{L}$, and every word in $\mathcal{L}$ is a proper prefix of a word in $\mathcal{L}$ and also a proper suffix of a word in $\mathcal{L}$.

A shift space is a subset of $\mathcal{A}^{\mathbb{Z}}$ which is compact in the product of the discrete topology on $\mathcal{A}$ and invariant under the shift homeomorphism $\sigma$, defined for $\left(x_{i}\right)_{i=-\infty}^{\infty} \in \mathcal{A}^{\mathbb{Z}}$ by

$$
\sigma\left(\left(x_{i}\right)_{i=-\infty}^{\infty}\right)_{j}=x_{j+1} .
$$

The shift space $\Omega_{\mathcal{L}}$ generated by a language $\mathcal{L}$ is the set consisting of those bi-infinite sequences of $\mathcal{A}^{\mathbb{Z}}$ all of whose factors are in $\mathcal{L}$. Conversely, starting 
with a collection of sequences of $\mathcal{A}^{\mathbb{Z}}$, the set $\mathcal{L}$ of all factors of the sequences is a language and $\Omega_{\mathcal{L}}$ is the smallest shift space containing all the sequences.

A substitution $\varphi$ on $\mathcal{A}$ is a function from $\mathcal{A}$ to $\mathcal{A}^{*}$ taking symbols to nonempty words. One extends $\varphi$ to a map on words by concatenation; infinite words are taken to infinite words of the same type. The incidence matrix for the substitution $\varphi$ is the $\mathcal{A} \times \mathcal{A}$ array $A_{\varphi}$ with abth entry equal to the number of occurrences of symbol $a$ in $\varphi(b)$; as a matrix it is defined up to conjugation by a permutation matrix. A substitution is primitive if its incidence matrix is primitive, i.e., some positive integer power has all entries positive. The language of $\varphi$ consists of all words appearing in the words $\varphi^{n}(a), n \in \mathbb{N}$ and $a \in \mathcal{A}$. We write $\Omega_{\varphi}$ for the shift space generated by this language. Primitivity ensures that $\Omega_{\varphi}$ is a minimal uniquely ergodic shift space ([Mi74]). We say $\varphi$ is aperiodic if $\Omega_{\varphi}$ is infinite, for otherwise it consists of the translates of a periodic sequence. A substitution is unimodular if the determinant of its incidence matrix is \pm 1 . A substitution is of Pisot type if the characteristic polynomial of its incidence matrix is irreducible and has a single root of modulus $\geq 1$. It is easy to see that a Pisot type substitution on an alphabet of at least two symbols is necessarily primitive and aperiodic.

A one-dimensional tiling space $\mathcal{T}$ is just the suspension of a shift space $\Omega$ under a function that is constant on each set of the form $[a]=\{x \in \Omega$ : $\left.x_{0}=a\right\}, a \in \mathcal{A}, \mathcal{A}$ is the alphabet of $\Omega$. Equivalently one may assign to each symbol in $\mathcal{A}$ a closed interval (a prototile) and then each sequence of $\Omega$ gives us a way of tiling of the real line by translates of prototiles. Denote by $\left(T_{t}\right)_{t \in \mathbb{R}}$ the natural $\mathbb{R}$-action on $\mathcal{T}$ by translation: $T_{t}$ moves the origin of a tiling $t$ units to the right. The topology on $\mathcal{T}$ is metrizable by a metric making two tilings close if and only if they agree on a large ball about the origin after a small translation. The metric is tedious to define (see [RS98]) but this characterization is all we shall need. The choice of lengths for the prototiles affects the conjugacy class of a tiling space but not the topology of the underlying space. If $\Omega$ is minimal and aperiodic then $\mathcal{T}$ is locally the product of a Cantor set and an arc ([Wil67]). Denote by $\mathcal{T}_{\varphi}$ the tiling space arising from the substitution $\varphi$.

For $\chi \in \mathcal{T}$, the composant of $\chi$ is the union of all proper subcontinua containing $\chi$. Composants in tiling spaces are always translation invariant. In one-dimensional tiling spaces, composants and arc-components are the same thing. We say two composants $X$ and $X^{\prime}$ of a one-dimensional tiling space $\mathcal{T}$ are forward [backward] asymptotic if there exist tilings $\chi \in X$ and $\chi^{\prime} \in X^{\prime}$ that are forward [backward] asymptotic under the flow. It is clear that asymptotic composants are nothing more than the arc-components of the suspensions of asymptotic symbolic sequences. Note that two symbolic 
sequences are forward [backward] asymptotic if and only they agree before [after] some point.

For the remainder of the paper we shall assume that our one-dimensional tiling spaces

(i) are aperiodic and minimal under translation,

(ii) have only finitely many asymptotic composants.

Remark. Primitive, aperiodic substitution tiling spaces satisfy these properties. See [GH55] for (i) and [Que87] for (ii).

Definition. Let $\xi=\left(\Delta_{1}, \ldots, \Delta_{r}\right)$ be a partition of the half-open interval $[0,1)$ into $r \geq 2$ half-open subintervals, numbered from left to right. Let $\pi$ be a permutation of $\{1, \ldots, r\}$. The interval exchange transformation, or IET for short, determined by $\xi$ and $\pi$ is the map $T:[0,1) \rightarrow[0,1)$ given by

$$
T(x)=x+\sum_{i: \pi(i)<\pi(j)}\left|\Delta_{i}\right| \quad \text { for } x \in \Delta_{j} .
$$

Interval exchanges preserve Lebesgue measure. We code symbolically orbits in an IET using the natural partition by the subintervals $\Delta_{i}$, and the corresponding symbolic interval exchange is the smallest shift space containing all the symbolic orbits. (The symbolic IET contains not only the codings of the left-continuous IET but also those of its right-continuous counterpart.)

We also need the notion of an interval exchange with reversals, a piecewise isometry just like an interval exchange except orientation reversing on some intervals. A precise definition requires special attention to the endpoints. We avoid this altogether by defining a symbolic interval exchange with reversals to be the shift space generated by the symbolic codings of orbits which do not meet endpoints.

Two maps are flow equivalent if there is a homeomorphism between their suspensions taking trajectories of one to trajectories of the other and preserving orientation.

3. Necessary conditions for embeddings. We first prove a theorem announced by Fedotov in [Fed80], stated here in the language of tiling spaces.

THEOREM 2. Let $\varphi$ be a primitive substitution with (aperiodic) tiling space $\mathcal{T}_{\varphi}$ and transition matrix $A_{\varphi}$. If $\mathcal{T}_{\varphi}$ embeds in a surface, then the product of the non-zero eigenvalues of $A_{\varphi}$ is \pm 1 .

Proof. The complement of the image of $\mathcal{T}_{\varphi}$ in the surface has only finitely many components (see Theorem 3 below). Let $\left(U_{i}\right)_{i \geq 1}$ be an increasing sequence of open sets such that: (i) each $U_{i}$ is a union of topological disks, with exactly one disk from each component of the complement and (ii) the union of the $U_{i}$ is the entire complement. The embedded continuum is the 
intersection of the decreasing sequence $\left(W_{i}\right)_{i \geq 1}$, where $W_{i}$ is the complement of $U_{i}$. Thus $\mathcal{T}_{\varphi}$ is homeomorphic to the inverse limit of the $W_{i}$ where the bonding maps are simply inclusion. Each of the $W_{i}$ has the same first cohomology, isomorphic to $\mathbb{Z}^{k}$ for some $k$, and the inclusions induce isomorphisms on cohomology. Since the cohomology of an inverse limit is the direct limit of the cohomologies, it follows that the first cohomology of $\mathcal{T}_{\varphi}$ is isomorphic to $\mathbb{Z}^{k}$. On the other hand, setting $d$ equal to the number of symbols in the alphabet for $\varphi$, we know from [AP98] that the first cohomology of the one-dimensional tiling space $\mathcal{T}_{\varphi}$ is isomorphic to the direct limit of

$$
\mathbb{Z}^{d} \underset{A_{\varphi}}{\longrightarrow} \mathbb{Z}^{d} \underset{A_{\varphi}}{\longrightarrow} \mathbb{Z}^{d} \longrightarrow \cdots .
$$

It is finitely generated if and only if the product of the nonzero eigenvalues of $A_{\varphi}$ is \pm 1 .

THEOREM 3. If $f: \mathcal{T} \rightarrow M$ is an embedding of a tiling space in a compact surface then the image of an arc-component of $\mathcal{T}$ is accessible from $M \backslash f(\mathcal{T})$ if and only if the arc-component is an asymptotic composant.

Before beginning the proof of the theorem, we first establish some notation and establish a lemma regarding the first return map of an arc transverse to $f(\mathcal{T})$. We will assume that $M$ is orientable. The nonorientable case is similar.

Let $\Lambda:=f(\mathcal{T}) \subset M$. Given $x \in \Lambda$, there exists a homeomorphism $\Psi$ : $(-1,1) \times(-1,1) \rightarrow U \subset M$ such that $\Psi((0,0))=x$ and $\Lambda \cap U=\Psi((-1,1) \times$ $\Sigma)$, where $\Sigma$ is a Cantor set in $(-1,1)$. Let $J$ be the $\operatorname{arc} \Psi(\{0\} \times(-1,1))$ and let $\Sigma^{\prime}=\Psi(\{0\} \times \Sigma)$. Thus, $\Sigma^{\prime}$ inherits an ordering from $\Sigma$ given by $y_{1}<y_{2}$ whenever $s_{1}<s_{2}$ for $y_{i}=\Psi\left(0, s_{i}\right)$.

For $y \in \Lambda$, let $y(t):=f\left(f^{-1}(y)-t\right)$. For each $y \in \Sigma^{\prime}$, there is a first return map $r(y) \in \Sigma^{\prime}$ given by $r(y)=y\left(t_{1}\right)$ where $t_{1}=\inf \left\{t>0: y(t) \in \Sigma^{\prime}\right\}$. For $y_{1}, y_{2} \in \Sigma^{\prime}$, let $D\left(y_{1}, y_{2}\right)=\left|s_{1}-s_{2}\right|$. Also, whenever $y_{1}<y_{2}$, define $\left[y_{1}, y_{2}\right]=\left\{y \in \Sigma^{\prime}: y_{1} \leq y \leq y_{2}\right\}$.

Lemma 3.1. There exists an $\varepsilon>0$ such that if $y_{1}<y_{2} \in \Sigma^{\prime}$ and $D\left(y_{1}, y_{2}\right)<\varepsilon$, then the first return $r:\left[y_{1}, y_{2}\right] \rightarrow\left[r\left(y_{1}\right), r\left(y_{2}\right)\right]$ is an order preserving homeomorphism.

Proof. The lemma is a consequence of the compactness of $\Sigma^{\prime}$ and the "Lemma of the Long Box" of Aarts and Martens ([AM88]; see also [FO96, Lemma 5.2]).

Given $x, y \in \Lambda$ lying on the same arc-component of $\Lambda$, there are homeomorphisms $\Psi_{x}$ and $\Psi_{y}$ as above establishing local neighborhoods of $x$ and $y$, respectively that are images of an open interval cross a Cantor set. The lemma of Aarts and Martens establishes a "long box" (a homeomorphic image of an open interval and a Cantor set) that is a neighborhood of the 
entire arc connecting $x$ to $y$. Given $x \in \Sigma^{\prime}$, we apply this lemma to $x$ and $r(x)$ and note that this "long box" neighborhood may have very small width that depends on the choice of $x$. Let $\delta_{x}$ be the width of this neighborhood in $\Sigma^{\prime}$. We thus have induced a collection of open intervals around each $x$ in $\Sigma^{\prime}$ of width $\delta_{x}$ that we take as a cover of $\Sigma^{\prime}$. The return map on each of the intervals is a homeomorphism onto its image that must preserve order since it is defined by the flow through the "long box" $N$. The Lebesgue Number Lemma then provides the desired $\varepsilon$.

REMARK. The lemma essentially says we can induce a map on $\Sigma^{\prime}$ that is a piecewise order preserving homeomorphism. If two points are within $\varepsilon$, then they must be in the same piece and are in fact associated to homotopic $\operatorname{arcs}$ in $M$. It should also be pointed out that should $M$ not be orientable, some pieces will be orientation reversing homeomorphisms. The following proof can be easily modified to include this possibility.

Proof of Theorem 3. We can now begin with the proof of the theorem. The complement of $\Lambda$ can be divided into disks, each of which is bounded by $\Lambda$. Let $V$ be one such disk and $\widetilde{V}$ be the prime end compactification of $V$. Let $y \in \Lambda$ be accessible from $V$. Then $y(t)$ is accessible from $V$ for all $t \in \mathbb{R}$. Let $z(t) \in \partial \widetilde{V}$ be the prime end corresponding to $y(t)$. Then $\{z(t): t \in \mathbb{R}\}$ is an open arc on the circle $\partial \widetilde{V}$. Let $z_{\infty}:=\lim _{t \rightarrow \infty} z(t)$ be one of the endpoints of this arc.

Fixing a point $x \in \Lambda$, we may choose a chain $\left\{C_{n}\right\}_{n=1}^{\infty}$ of cross-cuts representing the prime end $z_{\infty}$ with the following properties:

(i) $C_{n}$ is a closed arc with one endpoint $y_{n}=y\left(t_{n}\right)$ for $t_{1}<t_{2}<\cdots$,

(ii) $C_{n} \rightarrow x$ in the Hausdorff metric.

Now, let $\Psi:(-1,1) \times(-1,1) \rightarrow U$ be as above with $U$ a neighborhood of $x$. Then $C_{n} \rightarrow x$ implies that $C_{n} \subset U$ for sufficiently large $n$ (so we assume this happens for all $n)$. As $\Psi^{-1}\left(C_{n}\right)$ is an arc from $\left(a_{n}, s_{n}\right) \in(-1,1) \times \Sigma$ to $\left(a_{n}^{\prime}, s_{n}^{\prime}\right) \in(-1,1) \times \Sigma$ with $s_{n} \neq s_{n}^{\prime}$, and otherwise missing $(-1,1) \times \Sigma$, we may replace $C_{n}$ by the cross-cut $\Psi\left(C_{n}^{\prime}\right)$ with $C_{n}^{\prime}=\{0\} \times\left[s_{n}, s_{n}^{\prime}\right]$. Further, the chain $\left\{\Psi\left(C_{n}^{\prime}\right)\right\}_{n=0}^{\infty}$ of cross-cuts also represents $z_{\infty}$ and satisfies conditions (i) and (ii) above.

So we take $C_{n}=\Psi\left(C_{n}^{\prime}\right)=\Psi\left(\{0\} \times\left[s_{n}, s_{n}^{\prime}\right]\right)$ and either $y_{n}=\Psi\left(\left(0, s_{n}\right)\right)$ or $y_{n}=\Psi\left(\left(0, s_{n}^{\prime}\right)\right)$. We note that the points $s_{n}$ and $s_{n}^{\prime}$ are adjacent endpoints in the Cantor set and $\left|s_{n}-s_{n}^{\prime}\right| \rightarrow 0$.

Let $w_{1}=y_{1}$ and let $w_{n+1}=r^{n}\left(w_{1}\right)$ for $n=1,2, \ldots$ Then there exists an increasing sequence $k_{n}$ so that $y_{n}=w_{k_{n}}$. Let $v_{n}$ be the endpoint of $\Sigma^{\prime}$ adjacent to $w_{n}$. Thus there exists an $N$ such that for all $n \geq N,\left|p_{n}-q_{n}\right|<\varepsilon$ for $p_{n}, q_{n} \in \Sigma$ with $\Psi\left(\left(0, p_{n}\right)\right)=w_{n}$ and $\Psi\left(\left(0, q_{n}\right)\right)$. Then by Lemma 3.1, $r\left(v_{n}\right)=v_{n+1}$ for all $n$. 

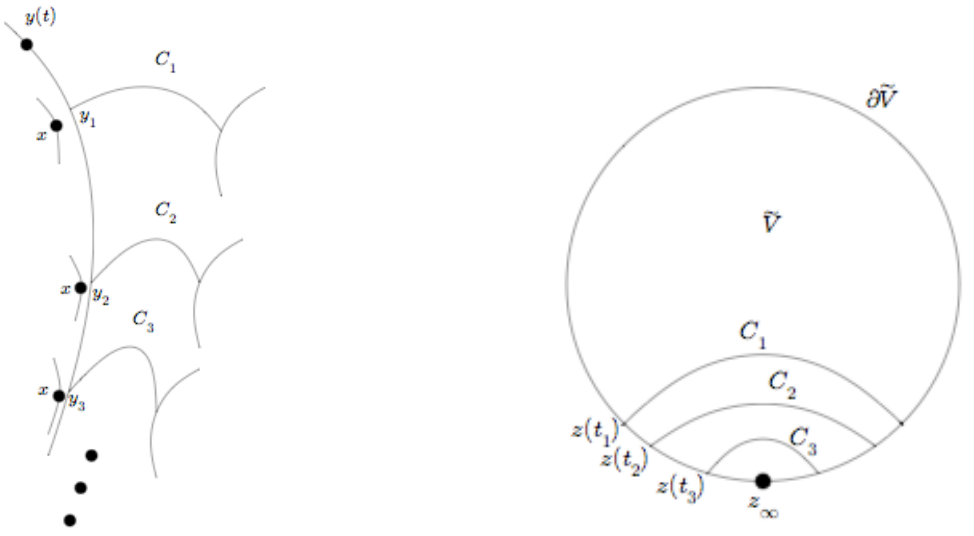

Fig. 1. Left: Cross-cuts representing $z_{\infty}$. One endpoint of each $C_{n}$ is on $y$. A priori, we do not know the other endpoints land on a single arc-component, as will be shown. Minimality and the decreasing length of the $C_{n}$ allow us to choose $y_{n}$ so that $C_{n} \rightarrow x$. Right: The circle of prime ends.

We have thus shown that all the cross-cuts $C_{n}$ are landing on the same arc-component on the end opposite $y_{n}$. We must now show that we can parametrize the arc-component associated to $y$ and $v$ so that they are asymptotic.

Let $t_{n}$ and $t_{n}^{\prime}$ be such that $w_{n}\left(t_{n+1}-t_{n}\right)=w_{n+1}$ and $v_{n}\left(t_{n+1}^{\prime}-t_{n}^{\prime}\right)=v_{n+1}$. Then $\left|t_{n+1}-t_{n}\right|$ and $\left|t_{n+1}^{\prime}-t_{n}^{\prime}\right|$ are uniformly bounded due to the uniform recurrence of the flow in $\mathcal{T}$. Further, $\left|t_{n}-t_{n}^{\prime}\right| \rightarrow 0$ since $D\left(w_{n}, v_{n}\right) \rightarrow 0$. It then follows that the rays $w(t)$ and $v(t)$ for $t \in \mathbb{R}^{+}$can be reparametrized so as to be asymptotic, which in turn implies that the composants $f^{-1}(y)$ and $f^{-1}\left(v_{1}(0)\right)$ are forward asymptotic. A similar argument could be made for finding a composant backward asymptotic to $f^{-1}(y)$.

For the converse of the theorem, we show that any asymptotic composant is accessible. Consider a pair of forward asymptotic composants, $X_{1}$ and $X_{2}$. We may parametrize $\mathcal{X}_{1}=f\left(X_{1}\right)$ and $\mathcal{X}_{2}=f\left(X_{2}\right)$ such that $D\left(y_{1}(t), y_{2}(t)\right)<\varepsilon$ for all $t \geq 0$. Let $y_{1}=y_{1}(0)$ and $y_{2}=y_{2}(0)$. We can once again use Lemma 3.1, to make a long box that connects $y_{i}$ to $r\left(y_{i}\right)$ for $i=1,2$. Note that $D\left(r\left(y_{1}\right), r\left(y_{2}\right)\right)$ is also less than $\varepsilon$ so that there is a transition into another long box. Any arc-component, $\mathcal{X}^{\prime}=f\left(X^{\prime}\right)$, of $f(\mathcal{T})$ that then enters this box between $y_{1}$ and $y_{2}$ is thus trapped between $\mathcal{X}_{1}$ and $\mathcal{X}_{2}$. Hence $X^{\prime}$ is forward asymptotic to $X_{1}$ and $X_{2}$. But there are only finitely many asymptotic composants and therefore $X^{\prime}$ would be isolated from the rest of $f(\mathcal{T})$. It would thus be accessible from both sides, contradicting minimality. Therefore $X_{1}$ and $X_{2}$ must be adjacent and hence accessible. 
COROLlary 3.2. If $\mathcal{T}_{\varphi}$ embeds in a surface, then the asymptotic composants must form $n$-cycles, where $n$ is an even integer.

Theorem 2 and Corollary 3.2 give us useful and computable necessary conditions for a tiling space to embed in a surface. The conditions are not sufficient. In a forthcoming work of Barge and the second author, it is shown the substitution tiling space generated by

$$
1 \mapsto 1131, \quad 2 \mapsto 1231, \quad 3 \mapsto 232
$$

satisfies both conditions but does not embed in any surface.

4. General results. Recall that an embedding $f: X \rightarrow Y$ is called essential if any continuous $g: Y \rightarrow Z$ is an embedding whenever $g \circ f$ is an embedding. In our setting, an embedding of a tiling space in a surface is essential if the image of each cycle of asymptotics bounds a disk.

TheOREM 4. Let $\mathcal{T}$ be a tiling space that embeds in a compact surface $M$. Then $\mathcal{T}$ embeds essentially in some closed surface $\widetilde{M}$ and extends to a foliation with singularities of all of $\widetilde{M}$.

Proof. The proof closely follows [FO96] and we use much of their notation. We may regard $\mathcal{T}$ as a subset of $M$, a lamination in the sense of [FO96]. Corollary 3.2 states that the complementary regions are bounded by the asymptotics. We may then remove any excess handles in these regions so that each cycle of asymptotics bounds a disk. We refer to this new manifold as $\widetilde{M}$, and again consider $\mathcal{T}$ to be a subset of it.

Since $\mathcal{T}$ is locally the product of a Cantor set and an arc, we may take a small matchbox neighborhood of a point of $\mathcal{T}$ in $\widetilde{M}$. Since this neighborhood must embed tamely into the plane (see Theorem 6.2 of [FO96], or [TW98]), we may find a transverse arc to the matchbox and pull it back to $\widetilde{M}$ so that it is transverse to the lamination $\mathcal{T}$ on $\widetilde{M}$. Let $K$ be this transverse arc. We may assume that the endpoints of $K$ are not in $\mathcal{T}$. We may also assume that $K$ was chosen small enough so that the orientations inherited from the tiling space of each arc-component crossing $K$ are in agreement. By minimality, $K$ intersects each arc-component of $\mathcal{T}$ and $K \cap \mathcal{T}$ is a Cantor set. Each arc of $\mathcal{T} \backslash K$ has two endpoints in $K$.

Let the endpoints of $K$ be denoted by $a$ and $b$. We form the double of $K$ on the surface $\widetilde{M}$ by taking two copies of $K$ denoted by $K \times\{+,-\}$ and identifying $(a,-)$ with $(a,+)$ and $(b,-)$ with $(b,+)$. Form a surface (with boundary) $\widehat{M}$ by opening up $\widetilde{M} \backslash K$ along the arc $K$ and compactifying using the double of $K$. Each $\operatorname{arc}$ of $\mathcal{T} \backslash K$ has one end on each side of the double of $K$, and we may assume that the arcs are oriented from $K \times\{+\}$ towards $K \times\{-\}$. 
We consider two arcs $L_{1}$ and $L_{2}$ in $\mathcal{T} \cap \widehat{M}$ to be homotopic if there exists a homotopy of $\widehat{M}$ which moves $L_{1}$ to $L_{2}$ and leaves each side of the double of $K$ invariant. By compactness, there are finitely many homotopy types and if two arcs are sufficiently close then they are homotopic. Therefore $K \times\{+\}$ can be divided into finitely many nonoverlapping intervals $I_{1}, \ldots, I_{n}$ such that two arcs are homotopic if they have an endpoint in the same interval. Because these arcs come from a tiling space, we may view them as being marked by tiles; nearby arcs must be marked identically. We may thus assume $I_{1}, \ldots, I_{n}$ are chosen so that any two arcs beginning in the same subinterval are marked the same. See the remark following the proof of Theorem 1 below.

For $l=1, \ldots, n$, let $\mathscr{A}_{l}$ be the set of $\operatorname{arcs}$ in $\mathcal{T} \cap \widehat{M}$ with left endpoint in $I_{l}$. Let $J_{l}$ be the smallest interval in $K \times\{-\}$ that contains all of the opposite endpoints of arcs in $\mathscr{A}_{l}$. Since all arcs in $\mathscr{A}_{l}$ are homotopic, there exist two arcs $\gamma_{l, 1}$ and $\gamma_{l, 2}$ in $\widehat{M}$ (not necessarily in $\mathcal{T}$ ), each homotopic to the arcs of $\mathscr{A}_{l}$, joining the endpoints of $I_{l}$ with the endpoints of $J_{l}$ in such a way that $\gamma_{l, 1} \cup \gamma_{l, 2} \cup I_{l} \cup J_{l}$ is a simple closed curve bounding a domain containing $\mathscr{A}_{l} \backslash \bigcup_{i \neq l} \mathscr{A}_{i}$. We call this domain $S_{l}$ a strip. We may now map $\gamma_{l, 1} \cup \gamma_{l, 2} \cup I_{l} \cup J_{l}$ to a rectangle laminated by arcs of $\mathscr{A}_{l}$. We again use the fact that a Cantor set cross an arc tamely embeds in the plane (again by Theorem 6.2 of [FO96] or [TW98]) to extend this lamination of the rectangle by a Cantor set of purely horizontal lines to a foliation of the rectangle. We now pull this foliation back to $\widetilde{M}$ to obtain a foliation of $S_{l}$.

Thus the lamination $\mathcal{T}$ of $\widehat{M}$ can be written as the union of strips on each of which we may extend the lamination to a foliation. Further, we may collapse the complementary domains of $\bigcup_{i} S_{i}$ to obtain a foliation of $\widehat{M}$ with singularities. This collapsing is possible once again by Corollary 3.2. Finally, we identify the appropriate points of $K^{+}$and $K^{-}$on the double of $K$ to recover $K$ and hence obtain a foliation of $\widetilde{M}$.

Note that an essential embedding guarantees that each cycle of asymptotics bounds a disk in the surface. Thus, the asymptotic composants completely determine the surface. We can assume that our embedding is smooth and extend the flow induced by the embedding to all of $\widetilde{M}$, with an $n$ pronged singularity in the component of the complement bounded by a cycle of $n$ asymptotics. The index of such a singularity is $-(n-2) / 2$. The Poincaré-Hopf Index Theorem then identifies the surface:

Proposition 4.1. Let $\mathcal{T}$ be a tiling space that embeds essentially in a compact surface $\widetilde{M}$. Then the Euler characteristic of $\widetilde{M}$ equals the sum of the indices of the cycles of asymptotics for $\mathcal{T}$. 
Proof of Theorem 1. A periodic tiling space is a circle and trivially embeds in the two-sphere and corresponds to an interval exchange on one interval. We may therefore assume that our tiling space is aperiodic.

The forward implication is trivial. Starting with a minimal interval exchange transformation, we can form the suspension, connecting each subinterval to its image with a strip. The boundaries of these strips form closed curves and they can be capped off with disks to form a closed surface. Indeed, since an IET has only finitely many asymptotic composants, we can say exactly what surface we get using index theory.

The converse is also straightforward. We use the notation from the proof of Theorem 4 above. The transverse measure on $K \cap \mathcal{T}$ induced by any ergodic measure on $\mathcal{T}$ is preserved by the first return map to $K \cap \mathcal{T}$. Reparametrize so that the width of each strip $S_{l}$ is the transverse measure of $I_{l} \cap \mathcal{T}$.

If $\widetilde{M}$ is orientable then the first return is order-preserving, and this gives us a symbolic interval exchange whose suspension is easily seen to be homeomorphic to $\mathcal{T}$. If $\widetilde{M}$ is not orientable then some of the intervals return with orientation reversed, and we have a symbolic interval exchange with reversal.

REMARK. The further reduction of our partition in the proof of Theorem 4 to arcs that are marked identically by tiles is necessary to avoid losing information. If our interval exchange has two adjacent intervals that map to adjacent intervals, then the corresponding paths through the surface are homotopic and our construction may otherwise glue them together as one interval. Example of this occurring are easy to generate by "peeling apart" a single tiling to obtain an additional 2-cycle. For example, a derived-from-Anosov map on the torus is obtained by peeling apart the unstable manifold of a periodic point (actually replacing a saddle periodic point with a small neighborhood containing a repelling point). One can then repeat this procedure by peeling apart the unstable manifold of a different periodic point. Two complementary domains would thus be present and each is bounded by a 2-cycle. The interval exchange, on any transverse arc, however, would always have two adjacent intervals mapped to adjacent intervals. Such a "doubly" derived-from-Anosov map on the torus is described by $1 \mapsto 1132,2 \mapsto 132,3 \mapsto 1332$, which exhibits this phenomenon. The fixed tilings given by ...1132 ... and ...1332 ... form a two-cycle and correspond to homotopic leaves in the surface. Hence paths marked by 1 and 3 are homotopic, and if we do not distinguish them then the resulting interval exchange corresponds to $1 \mapsto 1112,2 \mapsto 112$. The sliding block code $1 \mapsto 1,2 \mapsto 2,3 \mapsto 1$ defines a factor map from the first shift space to the second, that is one-to-one except on a single orbit, where it is two-toone. 
5. Substitutions on two symbols. In this section, we restrict our attention to substitutions on two letters and show that the property of embedding in a surface is related to being Sturmian (defined below). For an excellent introduction to Sturmian sequences and their role in substitutions, see Chapter 6 of [Fog01]. In what follows we use the notation and definitions from [Lot02] and [Fog01].

The complexity function $p(n)$ of a sequence assigns to each integer $n$ the number of distinct subwords of length $n$. If a sequence is not periodic, then $p(n) \geq n+1$ for all $n$. A sequence is called Sturmian if $p(n)=n+1$. In that sense Sturmian sequences have the smallest complexity among aperiodic sequences. A condition equivalent to being Sturmian is to be an aperiodic balanced sequence over two letters. A sequence over two letters is balanced if any two words of the same length differ in their abelianization in at most one place. In what follows, we will use the fact that a Sturmian sequence must have 00 or 11 as an allowed word, but not both.

Proposition 5.1. The asymptotic composants of a unimodular Pisot substitution on two symbols:

(1) form a single two-cycle if and only if the substitution is Sturmian,

(2) form a single four-cycle if and only if the substitution has four periodic points,

(3) do not form cycles in all other cases.

Proof. Let $\varphi$ be a unimodular Pisot substitution on $\mathcal{A}=\{0,1\}$ whose asymptotic composants form cycles. By the result of [BDH03], $\varphi$ has at most four asymptotic composants, so the cycles could conceivably be a single two cycle, a pair of two-cycles, or a single four-cycle. It is known that if $\varphi$ is Sturmian then its asymptotics form a single two-cycle.

Let us describe a simple method for finding the asymptotics for $\varphi$. Observe that if $\varphi(0)$ and $\varphi(1)$ both begin in symbol $a$, i.e.,

$$
\varphi(0)=a W^{(0)} \quad \text { and } \quad \varphi(1)=a W^{(1)},
$$

then the substitution

$$
0 \mapsto W^{(0)} a, \quad 1 \mapsto W^{(1)} a
$$

generates the same tiling space. Thus, by repeated application of this trick, and then replacing the result by its second iterate, we can assume

$(\dagger) \varphi(0)$ begins in 0 and $\varphi(1)$ begins in 1 ,

$(\dagger \dagger)$ all periodic points of $\varphi$ in $\{0,1\}^{\mathbb{Z}}$ are fixed points.

This completely solves the suffix problem (see [BDH03]) and it follows that the backward asymptotic pairs are

$$
\varphi^{\infty}(0) \cdot \varphi^{\infty}(0) \text { and } \varphi^{\infty}(0) \cdot \varphi^{\infty}(1)
$$


if $\varphi(0)$ ends in 0 and 00 is in the language of $\varphi$, and

$$
\varphi^{\infty}(1) \cdot \varphi^{\infty}(0) \text { and } \varphi^{\infty}(1) \cdot \varphi^{\infty}(1)
$$

if $\varphi(1)$ ends in 1 and 11 is in the language of $\varphi$. One or both of these must happen; both happen if and only if $\varphi$ has four fixed points, in which case we have the four-cycle as claimed. In the other case we have only one backward asymptotic pair; by interchanging the roles of 0 and 1 we may assume that it is the first one above. Since the asymptotics form cycles, some translates of $\varphi^{\infty}(0) \cdot \varphi^{\infty}(0)$ and $\varphi^{\infty}(0) \cdot \varphi^{\infty}(1)$ are forward asymptotic. Let $U$ be the left-infinite word $\varphi^{\infty}(0)$. There exist nonempty finite words $W^{(0)}, W^{(1)}$, beginning in different symbols and ending in different symbols, and a right-infinite word $V$ such that

$$
\varphi^{\infty}(0) \cdot \varphi^{\infty}(0)=U \cdot W^{(0)} V \text { and } \varphi^{\infty}(0) \cdot \varphi^{\infty}(1)=U \cdot W^{(1)} V .
$$

These are both fixed points of $\varphi$ and it follows from this and unimodularity and aperiodicity that $W^{(0)}$ and $W^{(1)}$ have the same abelianization.

We shall use two combinatorial facts about unimodular Pisot substitutions on two symbols satisfying $(\dagger)$ and $(\dagger \dagger)$ above. The proofs are elementary, and we omit them.

(1) The longest common suffix of $\varphi(01)$ and $\varphi(10)$ has length less than or equal to $|\varphi(01)|-2$, and if equality holds then $\varphi$ is Sturmian.

(2) If two words in $\mathcal{A}^{-\mathbb{N}}$ end in different symbols then the longest common suffix of their images under $\varphi$ is the longest common suffix of $\varphi(01)$ and $\varphi(10)$.

Applying the second fact to $U W^{(0)}$ and $U W^{(1)}$ we find that $\varphi\left(W^{(i)}\right)=$ $W^{(i)} V^{\prime}, i=0,1$, where $V^{\prime}$ is a prefix of $V$ and also a suffix of $\varphi(01)$ of length $\leq|\varphi(01)|-2$. Let $K$ be the word such that $K V^{\prime}=\varphi(01)$. Note that $K$ must contain both a 0 and a 1 . Taking $h$ to be the abelianization function for words, we obtain

$$
A_{\varphi}\left(h\left(W^{(0)}\right)\right)=h\left(W^{(0)}\right)+A_{\varphi} h(01)-h(K),
$$

or equivalently,

$$
A_{\varphi}\left(h\left(W^{(0)}\right)-h(01)\right)=h\left(W^{(0)}\right)-h(K) .
$$

Thus $h\left(W^{(0)}\right)-h(01)$ has nonnegative entries and

$$
A_{\varphi}\left(h\left(W^{(0)}\right)-h(01)\right) \leq h\left(W^{(0)}\right)-h(01),
$$

which can only happen if $h\left(W^{(0)}\right)=h(01)$. By the first fact, $\varphi$ is Sturmian and the proof is complete.

Sturmian spaces are the codings of 2-IETs with respect to their natural partitions and as such can be embedded in the torus $\mathbb{T}^{2}$. The four-cycle case cannot embed in an orientable surface since it would have to embed 
essentially in a surface of Euler characteristic -1 by Proposition 4.1. The other cases do not embed in any compact surface, by Corollary 3.2. We therefore have:

Theorem 5. Let $\varphi$ be a Pisot substitution on two symbols. Then the following are equivalent.

(1) $\mathcal{T}_{\varphi}$ embeds in an orientable surface.

(2) $\mathcal{T}_{\varphi}$ embeds in a torus.

(3) $\Omega_{\varphi}$ is Sturmian.

Acknowledgements. We are thankful to Marcy Barge, Bob Williams, Lorenzo Sadun and Cameron Gordon for numerous useful suggestions and helpful discussions. We would also like to thank the referee for helping clarify many parts of the paper and especially for pointing out a flaw and some vague language in the proof of Theorem 3 .

\section{References}

[AM88] J. M. Aarts and M. Martens, Flows on one-dimensional spaces, Fund. Math. 131 (1988), 53-67.

[AP98] J. E. Anderson and I. F. Putnam, Topological invariants for substitution tilings and their associated $C^{*}$-algebras, Ergodic Theory Dynam. Systems 18 (1998), 509-537.

[BD01] M. Barge and B. Diamond, A complete invariant for the topology of onedimensional substitution tiling spaces, ibid. 21 (2001), 1333-1358.

[BDH03] M. Barge, B. Diamond, and C. Holton, Asymptotic orbits of primitive substitutions, Theoret. Comput. Sci. 301 (2003), 439-450.

[BH92] M. Bestvina and M. Handel, Train tracks and automorphisms of free groups, Ann. of Math. 135 (1992), 1-51.

[BC97] M. D. Boshernitzan and C. R. Carroll, An extension of Lagrange's theorem to interval exchange transformations over quadratic fields, J. Anal. Math. 72 (1997), 21-44.

[Bru05] H. Bruin, Asymptotic arc-components of unimodal inverse limit spaces, Topology Appl. 152 (2005), 182-200.

[CE03] T. Carlsen and S. Eilers, Augmenting dimension group invariants for substitution dynamics, Ergodic Theory Dynam. Systems 24 (2004), 1015-1039.

[CF02] A. Clark and R. Fokkink, Bihomogeneity of solenoids, Algebr. Geom. Topology 2 (2002), 1-9.

[Fed80] A. G. Fedotov, Williams solenoids and realization in 2-dimensional dynamical systems, Soviet Math. Dokl. 21 (1980), 835-839.

[FHR03] T. Fitzkee, K. Hockett and E. A. Robinson, A weakly mixing tiling dynamical system with a smooth model, in: Tilings of the Plane, Theoret. Comput. Sci. 303 (2003), 447-462.

[Fog01] N. P. Fogg (editors: V. Berthé, S. Ferenczi, C. Mauduit and A. Siegel), Substitutions in Dynamics, Arithmetics and Combinatorics, Lecture Notes in Math. 1794, Springer, 2002. 
[FO96] R. Fokkink and L. G. Oversteegen, The geometry of laminations, Fund. Math. 151 (1996), 196-207.

[GH55] W. Gottschalk and G. A. Hedlund, Topological Dynamics, Amer. Math. Soc. Colloq. Publ. 36, Amer. Math. Soc., Providence, RI, 1955.

[Lot02] M. Lothaire, Algebraic Combinatorics on Words, Cambridge Univ. Press, 2002.

[Mi74] P. Michel, Stricte ergodicité d'ensembles minimaux de substitution, C. R. Acad. Sci. Paris Sér. A 278 (1974), 811-813.

[RS98] C. Radin and L. Sadun, An algebraic invariant for substitution tiling systems, Geom. Dedicata 73 (1998), 21-37.

[Que87] M. Queffélec, Substitution Dynamical Systems-Spectral Analysis, Springer, Berlin, 1987.

[Rau79] G. Rauzy, Échanges d'intervalles et transformations induites, Acta Arith. 34 (1979), 315-328.

[TW98] E. D. Tymchatyn and R. Walker, Taming the Cantor fence, Topology Appl. 83 (1998), 45-52.

[Wil67] R. F. Williams, One-dimensional nonwandering sets, Topology 6 (1967), 473487.

[Wil70] -, Classification of one-dimensional attractors, in: Proc. Sympos. Pure Math. 14, Amer. Math. Soc., 1970, 341-361.

Department of Mathematics

The University of Texas at Austin

1 University Station/C1200

Austin, TX 78712, U.S.A.

E-mail: cholton@math.utexas.edu
Minnesota State University

Wissink 273

Mankato, MN 56001, U.S.A.

E-mail: martensen@mnsu.edu

Received 31 October 2006;

in revised form 15 May 2007 